\title{
Coupled noble gas-hydrocarbon evolution of the early Earth atmosphere upon solar UV irradiation
}

\author{
E. Hébrard ${ }^{\mathrm{a}, \mathrm{b}, \mathrm{c}, *}$, B. Marty ${ }^{\mathrm{a}}$ \\ ${ }^{a}$ CRPG-CNRS, Université de Lorraine, 15 rue Notre Dame des Pauvres, BP 20, 54501 Vandoeuvre lès Nancy Cedex, France \\ ${ }^{b}$ Université de Bordeaux, Laboratoire d'Astrophysique de Bordeaux, UMR 5804, F-33270 Floirac, France \\ ${ }^{c}$ CNRS, Laboratoire d'Astrophysique de Bordeaux, UMR 5804, F-33270, Floirac, France
}

\begin{abstract}
Using a new photochemical model of the Earth's early atmosphere, the relationship between noble gas photoionization and organic photochemistry has been investigated from the Archean eon to the present day. We have found that the enhanced UV emission of the young Sun triggered a peculiar atmospheric chemistry in a $\mathrm{CH}_{4}$-rich early atmosphere that resulted in the increased formation of an organic haze, similar to the preliminary results of a previous study (Ribas et al., 2010). We have investigated the interaction between this haze and noble gases photoionized by the UV light from the younger Sun. Laboratory experiments have shown indeed that ionized xenon trapping into organics (1) is more efficient that other ionized noble gases trapping and (2) results in a significant enrichment of heavy xenon isotopes relative to the light ones (e.g., Frick et al., 1979; Marrocchi et al., 2011). We find moreover preferential photoionization of xenon that peaks at an altitude range comparable to that of the organic haze formation, in contrast to other noble gases. Trapping and fractioning of ionized xenon in the organic haze could therefore have been far more efficient than for other noble gases, and could have been particularly effective throughout the Archean eon, since the UV irradiation flux from the young Sun was expected to be substantially higher than today (Ribas et al., 2010; Claire et al., 2012). Thus we suspect that the unique isotopic fractionation of atmospheric xenon and its elemental depletion in the atmosphere relative to other noble gases, compared to potential cosmochemical components, could have resulted from a preferential incorporation of the heaviest xenon isotopes into organics. A fraction of atmospheric xenon could have been continuously trapped in the forming haze and enriched in its heavy isotopes, while another fraction would have escaped from the atmopshere to space, with, or without isotope selection of the lightest isotopes. The combination of these two processes over long periods of time provides thereby a key process for explaining the evolution of its isotopic composition in the atmosphere over time that has been observed in Archean archives (Pujol et al., 2011).
\end{abstract}

Keywords: early Earth, young Sun, noble gases, photochemistry

\section{Introduction}

The Earth atmosphere evolved through time via extraterrestrial contributions, interactions with the solar and galactic cosmic ray irradiations and complex exchanges with the lithosphere, hydrosphere and, once arisen, biosphere. Understanding the state and evolution of the atmosphere during the Hadean and the Archean eons is of prime importance, most notably because Life evolved and flourished during this first half of Earth's history. Information about the atmosphere at such remote time is elusive owing to the lack of direct records, and the state and evolution of the atmosphere over Earth's history have been constrained for years from the geological record, no matter how blurred by metamorphism and rock alteration. There have been remarkable advances in the recent years and available information suggests nowadays that the ancient

*Tel: +33-5-5777-6124; fax: +33-5-5777-6110

Email address: hebrard@crpg.cnrs-nancy.fr (E. Hébrard) atmosphere was indeed very different from today. For instance, mass-independent isotopic fractionation of sulfur have been found in Archean sedimentary rocks, and is believed to be related to the occurrence of enhanced photochemical reactions induced by UV solar light at significant atmospheric depths (Farquhar et al., 2000). Likewise, the persistence of liquid water during the Archean eon, despite a faint young Sun, required certainly the presence of elevated concentrations of greenhouse gases in the early Earth's atmosphere (Sagan and Chyba, 1999; Feulner, 2012). Despite these studies, there is still considerable uncertainty concerning the origin and the evolution of the atmospheric volatiles themselves.

Noble gases are exceptional tracers of the sources and sinks of atmospheric volatiles, due to their chemical inertness and to contributions from nuclear reactions (e.g., Ozima and Podosek, 2002). Studies based on compositional differences between the present-day atmosphere and potential cosmochemical end-members (the meteorites, the solar nebula, the solar wind), and/or mantle-derived rocks 
(e.g., Ozima and Podosek, 2002; Pepin, 1991; Pepin and Porcelli, 2006) have provided important geochemical constraints. Firstly, the atmosphere contains noble gas isotopes produced by extant and extinct radioactive isotopes produced in the solid Earth, which permits investigation of mantle/crust degassing chronology. These tracers indicate that the atmosphere is a geologically old reservoir which formed early during accretion and differentiation of the Earth (Allègre et al., 1983; Zartman et al., 1961). Xenon presents an interesting discrepancy, known as the "xenon paradox". Firstly, this element is depleted by one order of magnitude relative to other nobles gases and other volatile elements (e.g. $\mathrm{H}\left({ }_{2} \mathrm{O}\right), \mathrm{C}$ ) when normalized to the chondritic composition (Marty, 2012, and references therein). Secondly, atmospheric xenon is largely enriched in its heavy isotopes relatively to potential extraterrestrial end-member compositions $\left(3-4 \% \mathrm{u}^{-1}\right)$, whereas other noble gas isotopic ratios resemble to those of potential cosmochemical ancestors (although atmospheric krypton is slightly fractionated too, by $<1 \% \mathrm{u}^{-1}$, relatively to its chondritic composition). Thirdly, atmospheric xenon corrected for $3-4 \% \mathrm{u}^{-1}$ isotope fractionation still differs from known chondritic or solar compositions suggesting that it originated from an extraterrestrial component having a specific composition. In order to circumvent the last point, Pepin (1991) postulated the existence of an adequate xenon ancestor that was subsequently isotopically fractionated to yield the composition of modern atmospheric xenon. However, mass-dependent fractionation from either an extraterrestrial reservoir during loss of noble gases from the atmosphere cannot account alone for both the elemental and isotopic of atmospheric xenon. Attemps to resolve this "xenon paradox", has led to sophisticated models of early atmospheric evolution coupled with mantle geodynamics (Pepin, 1991; Tolstikhin and Marty, 1998) and cometary contributions (Dauphas, 2003; Owen et al., 1992) that could explain terrestrial noble gas patterns under ad hoc conditions during the building stages of the Earth. Alternatively, it has been proposed that the xenon underabundance in the atmosphere could be due to its early sequestration in minerals at high pressures deep in the Earth (Sanloup et al., 2005; Shcheka and Keppler, 2012; Zhu et al., 2013; Sanloup et al., 2013), but this possibility alone cannot account for the specific xenon isotope composition of the Earth's atmosphere.

\section{Constraints from Archean noble gas isotopic com- position}

Recently, the analysis of Archean samples (3.5 Ga-old barite, Pujol et al. (2009); and hydrothermal quartz, Pujol et al. (2011, 2013)) permitted insight into the composition of noble gases in the Archean atmosphere. These samples have trapped several ancient fluids mixed up with a surface water component having dissolved noble gases, presumably from the Archean atmosphere. The isotopic

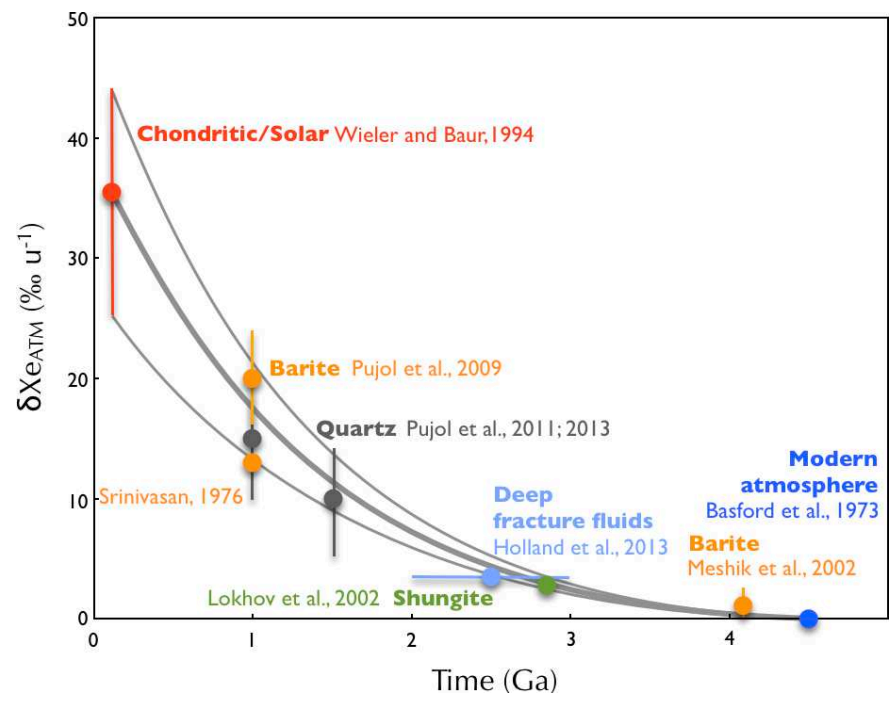

Figure 1: Proposed evolution of the xenon isotopic fractionation relative to the modern atmospheric composition, expressed in per mil per atomic mass unit $\left(\% \mathrm{u}^{-1}\right)$, as a function of time (adapted from Pujol et al. (2011)). Chondritic/solar value is the mean value of the solar and chondritic compositions (from Wieler and Baur (1994)). Barite and quartz samples are both from the Dresser formation at the North Pole area in Pilbara craton, Western Australia (Srinivasan, 1976; Pujol et al., 2009, 2011, 2013). Quartz samples are in fact fluid inclusions trapped in Archean hydrothermal quartz. The $1.65 \mathrm{Ga}-$ old shungite sample is from the Shun'ga area in the Karelia region, Russia (Lokhov et al., 2002). 2.0-3.0 Ga-old deep fracture fluid sample is from the Timmins mine in Ontario (Holland et al., 2013). The younger, 170 Ma-old, barite sample is from the Belorechenskoe deposit, Northern Caucasus, Russia (Meshik et al., 2001). The modern atmospheric ratio is from Basford et al. (1973). The data fit an exponential decay curve, suggesting that the xenon isotopic fractionation was progressive with time.

composition of xenon in these samples is intermediate between that of the modern atmosphere and that of potential cosmochemical ancestors. Furthermore, available data for xenon isotopes trapped in ancient rocks seem to define a temporal evolution (Fig. 1) suggesting that the xenon isotope composition of the atmosphere changed with time from a chondritic (or solar) one to the present-day one.

The isotopic composition of atmospheric xenon differs from that of the chondritic, or solar, compositions by a $3.5 \% \mathrm{u}^{-1}$ on average enrichment in its heavy isotopes relative to the light ones. Atmospheric xenon is elementally depleted relative to krypton by a factor of 23 on average, compared to carbonaceous chondrites (e.g., Pepin, 1991). We assume that these atmospheric depletion and isotope fractionation had a common cause and followed a Rayleigh distillation :

$$
\left(\frac{{ }^{i+1} \mathrm{Xe}}{{ }^{i} \mathrm{Xe}}\right)_{t} /\left(\frac{{ }^{i+1} \mathrm{Xe}}{{ }^{i} \mathrm{Xe}}\right)_{t=0}=f^{\alpha-1}
$$

where $\alpha$ is the fractionation factor during loss, $i$ the mass number of any stable xenon isotope and $f(=1 / 23)$ is the 
depletion factor of atmospheric xenon. Thus :

$$
\left(\frac{{ }^{i+1} \mathrm{Xe}}{{ }^{i} \mathrm{Xe}}\right)_{t=4.56 \mathrm{Ga}, \text { today }} /\left(\frac{{ }^{i+1} \mathrm{Xe}}{{ }^{i} \mathrm{Xe}}\right)_{t=0}=f^{\alpha-1} \sim 1.035
$$

The instantaneous fractionation factor $\alpha$ required for explaining the current enrichment in heavy isotopes for xenon remaining in the atmosphere is thus equal to $1.1 \%$ $\mathrm{u}^{-1}$. Processes able to fractionate the isotopes of xenon at such percent level require ionization of xenon (Frick et al., 1979; Bernatowicz and Fahey, 1986; Bernatowicz and Hagee, 1987; Ponganis et al., 1997; Hohenberg et al., 2002; Marrocchi et al., 2011). In experiments aimed at fractionating xenon isotopes upon ionization, ionized xenon is implanted into solids where it is enriched in heavy isotopes, whereas neutral xenon is much less efficiently trapped and is not isotopically fractionated.

Xenon depletion and isotope fractionation could have taken place progressively in the Archean atmosphere, through interaction with UV light from the young Sun. Compared to today, the irradiation flux was probably 5 times higher at $3.5 \mathrm{Ga}$ and 3 times higher at $2.5 \mathrm{Ga}$, based on observations of nearby solar type stars (Ribas et al., 2005). We suggest that most of the ionized xenon would have escaped while only a small fraction, implanted into atmospheric aerosols and enriched in heavy isotopes, would have been preserved. Such processing should have affected mostly xenon and not the other noble gases (although there is room for fractionating krypton to a much lesser extent), as developed in the present work. Over time, this combination of atmospheric escape and preservation of a tiny fraction of isotopically-fractionated xenon would have resulted in a Rayleigh-type distillation. Elemental and isotopic compositions of noble gases in general, and xenon in particular, may therefore reflect the chemical conditions of the early Earth's atmosphere.

It has been hypothesized that the Earth's atmosphere contained an organic haze similar to that of Titan well before widespread oxygenation about 2.45 Gyr ago (Kasting et al., 1983; Zahnle, 1986; Sagan and Chyba, 1999). This prediction arose from the fact that, because the Sun was fainter by $20-30 \%$ than today, and because there is no evidence in the geological record for a very high $\mathrm{P}_{\mathrm{CO}_{2}}$ in the Archean, another greenhouse gas had to be present in the ancient atmosphere to counter-balance the lower solar energy and prevent global glaciation (e.g., Kasting, 2010, and references therein). This prediction has been substantiated by geological evidence only recently. Pavlov et al. (2001b) have first argued that the occurence of kerogens with extremely low $-{ }^{13} \mathrm{C}$ in late Archean sediments constitutes a indirect evidence for the occurence of an organic haze at the time. Farquhar and Wing (2003) have used the sulfur isotope record to conclude that early to mid-Archean conditions were anoxic. The lifetime of methane in the early Earth's atmosphere was therefore of the order of $10^{3}$ to $10^{4}$ years in contrast to about 10 years in our modern atmosphere (Zahnle, 1986). Domagal-Goldman et al. (2008) have gone further by suggesting that the sulfur isotopic variability was modulated by the thickness of an organic haze. Zerkle et al. (2012) multiproxy geochemical analyses of sediments from the 2.65-2.5-billion-years-old Ghaap Group in South Africa, have indicated the presence at that time of a reduced atmosphere that was periodically rich in methane, consistent with the prediction of a hydrocarbon haze. Overall, as limited as they are, ground-truth evidences from 3.5 to 2.5-Ga-old geological records suggest that methane was indeed an important component of the atmosphere throughout the Archean, which might have driven the formation of a hydrocarbon haze.

Numerical simulations of the responses of organic haze formation and noble gases photoionization to the early Earth's atmosphere conditions and solar EUV variations are therefore worth performing. To evaluate these, we have developed a one-dimensional photochemical model of the primitive Earth's atmosphere in appropriate conditions for an organic haze to be formed and at different times along the course of the Sun's evolution. We explore conjointly the possible temporal evolution of the noble gas elemental and isotopic compositions of the atmosphere, due to the interaction with extreme UV light from the Sun.

\section{Model description}

Our photochemical model is derived from a recent model of the atmosphere of Titan (Hébrard et al., 2012), which has been updated on the basis of the latest model for the early Earth (Tian et al., 2011). The model now includes 83 species, for which transport is considered, linked by 351 chemical reactions. Instead of using a classical CrankNicholson method, we use the ODEPACK library, which implements Hindmarsh's solvers for ordinary differential equations (Hindmarsh, 1983).

Our one-dimensional photochemical model uses a constant background atmosphere. Atmospheric parameter inputs $(P, T, n)$ were taken from Tian et al. (2011). The corresponding temperature profile, without stratospheric heating and with a surface temperature of $273 \mathrm{~K}$, is at the lower limit of what is expected for the Archean, as evidenced by the presence of liquid water on the early Earth (see Feulner, 2012, for a complete review). The density profile is quite consistent with a recent study of fossil raindrop imprints that limits total air density at 2.7 Ga to less than twice today's level, with the most likely value similar to the present-day density (Som et al., 2012).

Previous theoretical studies have indicated that the rate of organic haze formation was a function of the atmospheric $\mathrm{CH}_{4} / \mathrm{CO}_{2}$ ratio (Pavlov et al., 2001a; Trainer et al., 2004; Kharecha et al., 2005). A high concentration of methane and a relatively low concentration of carbon dioxide tends to favor the production of an hydrocarbon haze both in theoretical models (Pavlov et al., 2001a) and in laboratory experiments (Trainer et al., 2006). Formation of hydrocarbon aerosols is calculated using methods similar to those used in previous works (Pavlov et al., 2001a; 


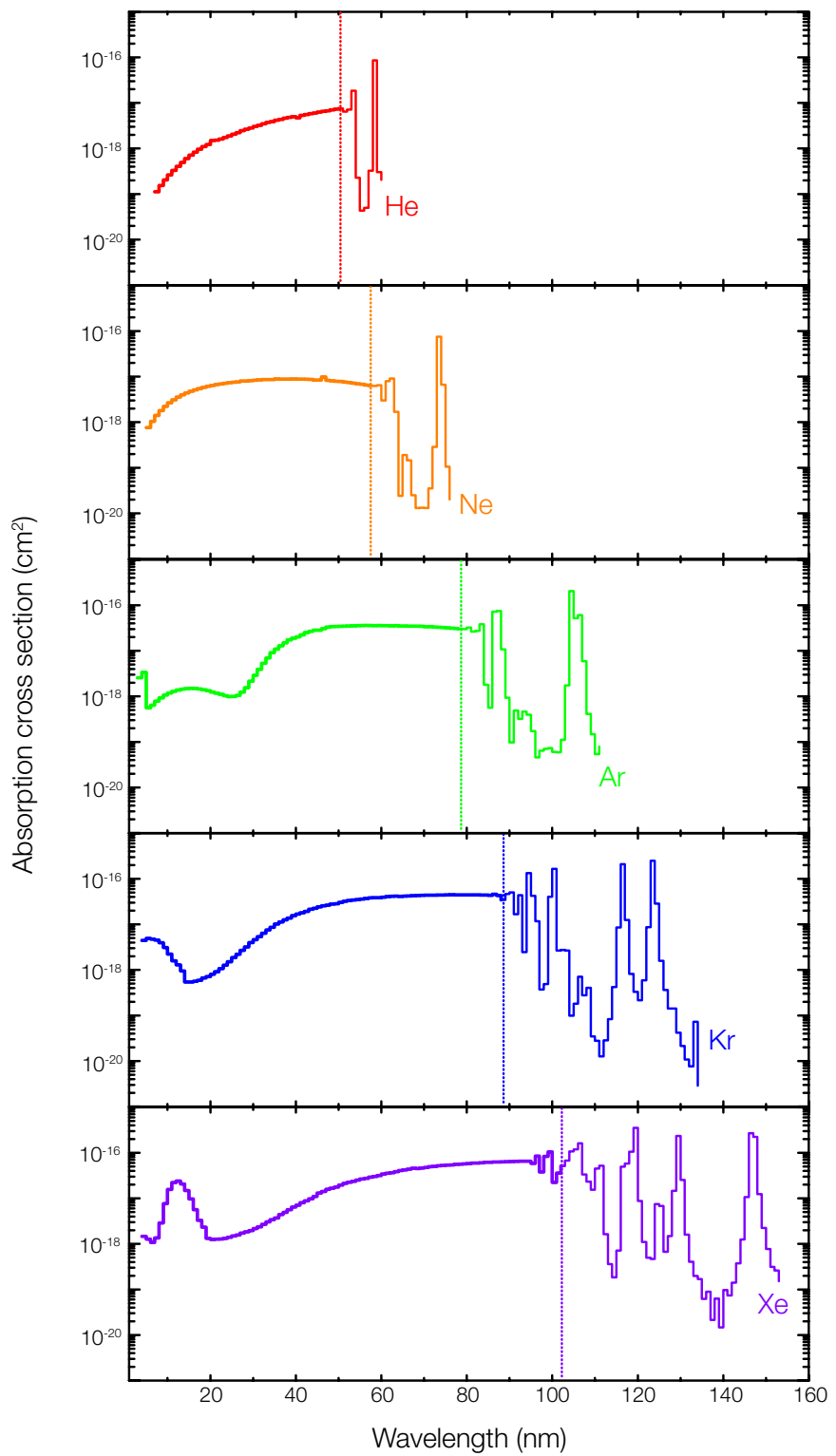

Figure 2: Absolute photoabsorption (plain lines) and photoionization (thick lines) cross sections of helium He, neon Ne, argon Ar, krypton $\mathrm{Kr}$ and xenon Xe binned at $1 \mathrm{~nm}$ resolution (adapted from Chan et al. (1991, 1992a,b)) along with their first ionization potentials (dashed lines) (Lide, 2009). Xenon has the lowest first ionization potential and the most extended photoabsorption range among noble gases.

Domagal-Goldman et al., 2008; Tian et al., 2011) but their absorption and scattering in the UV range are neglected. Hydrocarbon aerosols formation in our model relies therefore on the following reactions :

$$
\begin{gathered}
\mathrm{C}_{2} \mathrm{H}+\mathrm{C}_{2} \mathrm{H}_{2} \rightarrow \mathrm{C}_{4} \mathrm{H}_{2}+\mathrm{H} \\
\mathrm{C}_{2} \mathrm{H}+\mathrm{CH}_{2} \mathrm{CCH}_{2} \rightarrow \mathrm{H}+\mathrm{C}_{5} \mathrm{H}_{4}
\end{gathered}
$$

Truncating the polymerization scheme in this over-simplified way should maximize the formation of aerosol particles since no backward reactions for the resulting high molecular weight products are introduced. Following Pavlov et al. (2001a), we are here less interested in deriving the aerosols genuine composition than in finding a way to quantify simply the efficiency of the haze formation. To rely on an efficient haze formation in Earth's early atmosphere, we focus our calculations on the 1000 ppmv $\mathrm{CH}_{4}$ and 3000 ppmv $\mathrm{CO}_{2}$ case of Tian et al. (2011) model, which is applicable to the Archean postbiotic Earth (Rye et al., 1995; Kharecha et al., 2005; Rosing et al., 2010). Volcanic outgassing of sulfur dioxide $\mathrm{SO}_{2}$ and molecular hydrogen $\mathrm{H}_{2}$ is included. Their surface fluxes are set equal to $3.5 \times 10^{9}$ and $2.5 \times 10^{10}$ molecules $\mathrm{cm}^{-2} \mathrm{~s}^{-1}$, respectively (Pavlov and Kasting, 2002). A zero flux is assumed as an upper boundary condition for most of the species, except for atomic hydrogen $\mathrm{H}$ and molecular hydrogen $\mathrm{H}_{2}$, which are allowed to escape with velocities based on the diffusionlimited escape formula (Walker, 1977). This provides an upper limit on the escape rate, and hence a lower limit for their atmospheric abundances. The possibility of nonthermal escape of xenon will be discussed later on. The water vapor content in the troposphere is controlled by the temperature profile. Above the tropopause, the water vapor concentration is calculated by solving the combined equations of photochemistry and transport. The eddy diffusivity profile is taken from Massie and Hunten (1981). Calculations are performed with a solar zenith angle of $50^{\circ}$ to account for diurnally averaged conditions at the equator. We use a non-uniform grid of altitude with 125 levels from the ground to $150 \mathrm{~km}$. Two consecutive levels are separated by a distance smaller than $H(z) / 5$, where $H(z)$ is the atmospheric scale height at the altitude $z$.

Helium is lost continuously to space through thermal and non thermal processes, and is considered here only for the sake of illustration. The abundances of noble gas isotopes not produced by nuclear reactions and nor lost from the atmosphere, ${ }^{20} \mathrm{Ne}$ and ${ }^{84} \mathrm{Kr}$, are considered to be invariant through time. Pujol et al. (2013) recently reported the analysis of argon in 3.5-Ga-old hydrothermal quartz which revealed a ${ }^{40} \mathrm{Ar} /{ }^{36} \mathrm{Ar}$ ratio of $143 \pm 24$, lower than the present-day atmospheric value of $298.56 \pm 0.31$ (Lee et al., 2006). The evolution of atmospheric argon abundance through time was calculated by assuming that ${ }^{36} \mathrm{Ar}$ was primordial and by using the Archaean atmospheric ${ }^{40} \mathrm{Ar} /{ }^{36} \mathrm{Ar}$ ratios yielded by Pujol et al. (2013). The evolution of atmospheric xenon abundance through time was calculated by assuming that it follows a Rayleigh type isotope evolution. The xenon isotopic fractionation $\delta \mathrm{Xe}$ data displayed in Fig. 1 are fitted with an exponential decay curve :

$$
\delta \mathrm{Xe}=35.124 e^{-0.740 t}-1
$$

We consider that the instantaneous fractionation factor $\alpha$ did not vary through time. We calculate the depletion factor of atmospheric xenon $f$ through time as :

$$
\left(\frac{1+\frac{35.124 e^{-0.740 t}-1}{1000}}{1.035}\right)^{\frac{1}{\alpha-1}}
$$


The corresponding atmospheric abundances we derived are listed in Table 1.

The Sun was significantly more active in its past. This early activity for Sun-like stars has been shown in the Xray/EUV range (Ribas et al., 2005) and in the UV range (Ribas et al., 2010). These emissions might have had an impact on the early evolution of Earth's atmosphere. It has been proposed, for instance, that UV radiation might help the synthesis of complex ribonucleotides in plausible early Earth conditions (Powner et al., 2009). Through the use of a photochemical model, Ribas et al. (2010) indicate that such enhanced UV light emission leads indeed to a significant increase in photodissociation rates compared with those commonly assumed for the early Earth. These results emphasize the fact that reliable calculations of the physical state and the chemical composition of early planetary atmospheres need to account for the stronger solar photodissociating EUV-UV irradiation. As the solar emission in this wavelength range drives the photochemistry and thus the atomic and molecular compositions of planetary atmospheres, it might have impacted the formation of an organic haze as well as the photoionization of noble gases during the Archean. The high-resolution dipole (e,e) method has proven to be suitable for the measurement of absolute photoabsorption oscillator strengths (e.g. cross sections) for electronic excitation of free atoms and molecules and has been used therefore to derive the photoabsorption and photoionization cross sections for the different noble gases in the energy range 16-250 eV (Chan et al., 1991, 1992a,b). The specific electronic structure of xenon which makes it the most reactive element among noble gases is due to both its lowest ionization potential (12.13 eV or $102.23 \mathrm{~nm}$ (Lide, 2009)) and to its extended photoabsorption cross section covering part of the VUV spectrum (up to about $150 \mathrm{~nm}$ ).

We have run our one-dimensional photochemical model for the reducing Archean atmosphere using the modernday solar flux and fluxes for the Sun at $2.5 \mathrm{Ga}, 3.0 \mathrm{Ga}$ and $3.5 \mathrm{Ga}$. Claire et al. (2012) provided quantitative estimates of the wavelength dependence of the solar flux for periods of time relevant to the early evolution of planetary atmospheres in the Solar System or around other G-type stars. Fig. 3 displays solar actinic fluxes at 2.5, 3.0, and $3.5 \mathrm{Ga}$ along with the modern-day flux (Claire et al., 2012) and focuses on the specific emission-line behavior in the UV with inset showing the overall behavior with time of the whole solar spectrum.

Table 1: Noble gases atmospheric abundances through time (in ppmv).

\begin{tabular}{|c|c|c|c|c|}
\hline & Today & $2.5 \mathrm{Ga}$ & $3.0 \mathrm{Ga}$ & $3.5 \mathrm{Ga}$ \\
\hline $\mathrm{He}$ & 5.24 & $(5.24)$ & $(5.24)$ & $(5.24)$ \\
\hline $\mathrm{Ne}$ & & & & \\
\hline $\mathrm{Ar}$ & 9340 & 6889 & 5953 & 4488 \\
\hline $\begin{array}{l}\mathrm{Kr} \\
\mathrm{Xe}_{\mathrm{e}}\end{array}$ & 009 & 017 & 023 & 0.37 \\
\hline
\end{tabular}

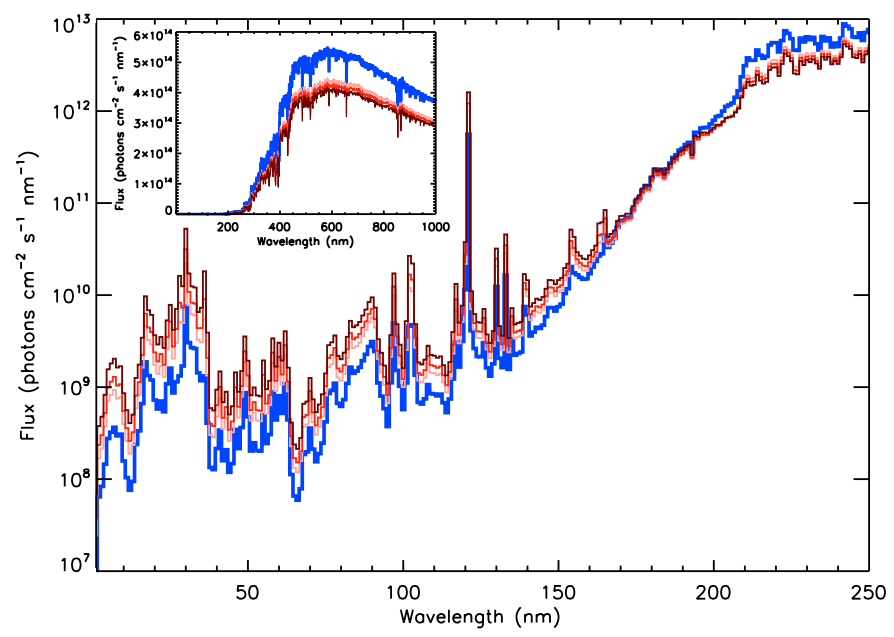

Figure 3: Predicted incoming solar flux at Earth vs. wavelength, at 3.5 Ga (dark red), 3.0 Ga (red) and 2.5 Ga (red), compared to the present solar flux (bold blue). Each panel displays the solar flux for the given paleodate, each binned at $1 \mathrm{~nm}$ resolution for comparison. In the main panel, the solar fluxes vs. wavelength are displayed on a logarithmic vertical scale in units of photons $\mathrm{cm}^{-2} \mathrm{~s}^{-1} \mathrm{~nm}^{-1}$ in the UV region of the spectrum driving the photochemistry, up to $250 \mathrm{~nm}$. The subpanel zooms out on the solar fluxes vs. wavelength, linearly displayed on most of the solar spectrum (adapted from Claire et al. (2012)).

\section{Results and discussion}

The fact to use a constant vertical temperature profile in this work is obviously not consistent with the evolution of insolation conditions through time. It would require to compute a vertical temperature profile at radiative equilibrium with a one-dimensional radiative-convective model. This is clearly beyond the scope of the present paper. This approximation has however little impact on the calculation of photolysis rates. Indeed, the purpose here is not to run a fully consistent radiative-convective-photochemical model, but to illustrate how the photoionization rates, the photodissociation rates and the subsequent photochemistry are sensitive to an enhanced UV irradiance in constant atmospheric conditions, all other things being kept equal.

\subsection{Temporal evolution of atmospheric chemistry}

We find that the atmospheric composition is very sensitive to the incoming solar flux that relates to the age of the Sun. The concentrations of important atmospheric species with corresponding rates for chemical production and loss reactions relevant to noble gases and haze photochemistry are displayed in Fig. 4 under the present Sun and in Figs. 5 under the Sun at $3.5 \mathrm{Ga}$. The most apparent differences in atmospheric gas concentrations are that: 1) methane $\mathrm{CH}_{4}$ and its photolysis derivatives (acetylene $\mathrm{C}_{2} \mathrm{H}_{2}$, ethylene $\mathrm{C}_{2} \mathrm{H}_{4}$, ethane $\mathrm{C}_{2} \mathrm{H}_{6}$ and propane $\mathrm{C}_{3} \mathrm{H}_{8}$ ) are less abundant with the Sun at $3.5 \mathrm{Ga}$ than with the present Sun (Figs. 4(a) and 5(a)); and 2) ionized noble gases are more abundant with the Sun at $3.5 \mathrm{Ga}$ than with the present Sun (Figs. 4(b) and 5(b)). 

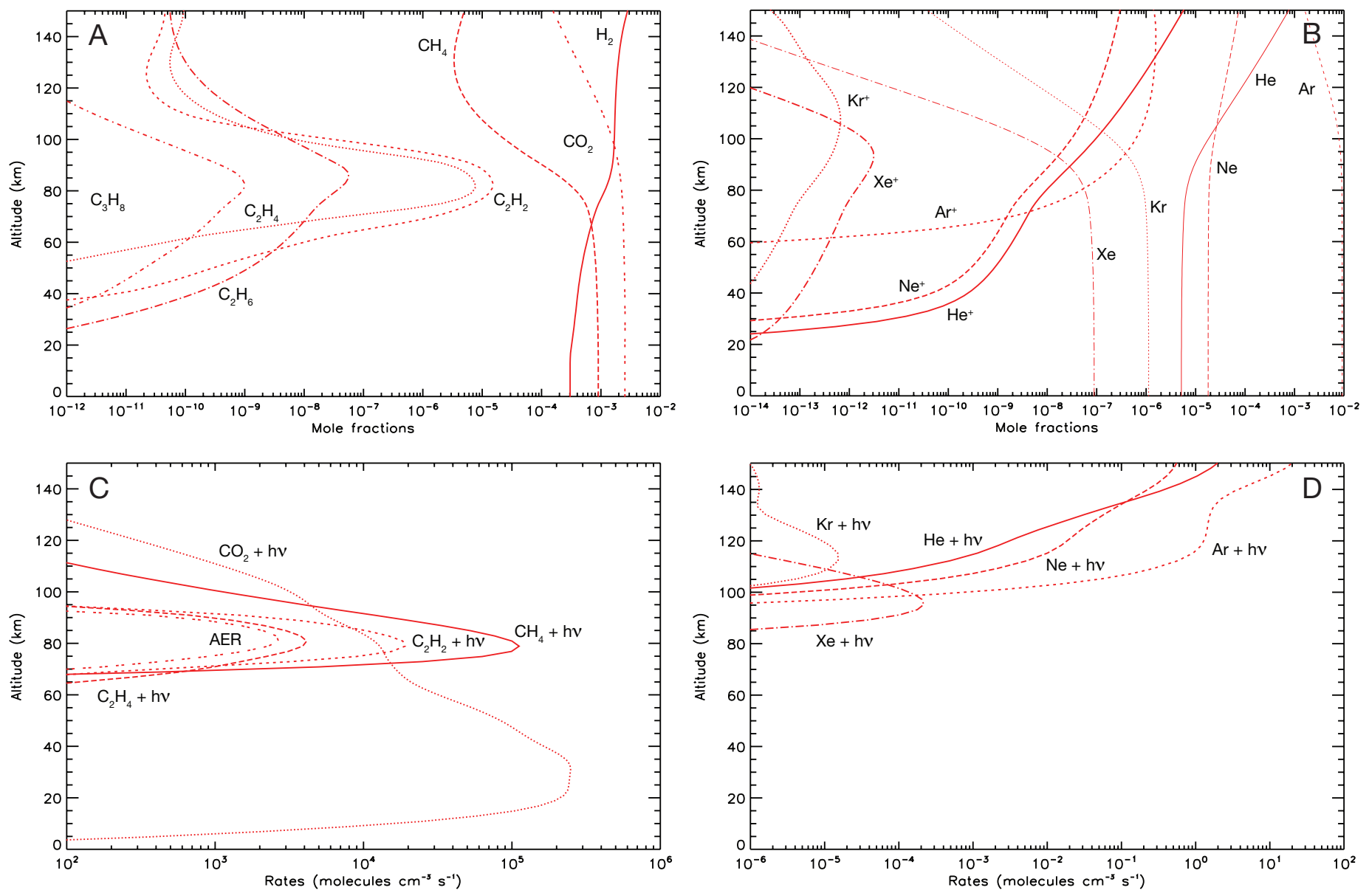

Figure 4: Results of the photochemical model with an incoming solar flux representative of the present Sun. Panel A displays the abundance profiles of molecular hydrogen $\mathrm{H}_{2}$, carbon dioxide $\mathrm{CO}_{2}$ and some key hydrocarbons. Panel B displays the abundance profiles of the neutral and photoionized noble gases. Panel $\mathrm{C}$ displays the photodissociation rates of carbon dioxide $\mathrm{CO}_{2}$ and some key hydrocarbons. The reaction AER mentioned in panel $\mathrm{C}$ is a combination of the following reactions: $\mathrm{C}_{2} \mathrm{H}+\mathrm{C}_{2} \mathrm{H}_{2} \rightarrow \mathrm{C}_{4} \mathrm{H}_{2}+\mathrm{H}$ and $\mathrm{C}_{2} \mathrm{H}+\mathrm{CH}_{2} \mathrm{CCH}_{2} \rightarrow \mathrm{H}+\mathrm{C}_{5} \mathrm{H}_{4}$. Panel $\mathrm{D}$ displays the photoionization rates of the noble gases. Please note the concordance between the altitude at which xenon photoionization peaks and the organic haze formation region.

The decrease of the abundances of methane and more complex hydrocarbons with the Sun at 3.5 Ga is consistent with the fact that their photolysis are their major loss processes in our modeled atmosphere, no matter what the age of the Sun is. As expected from previous studies (Ribas et al., 2010; Claire et al., 2012), the enhanced UV emission for the Sun at 3.5 Ga leads indeed to a significant increase in photodissociation rates compared with those of the modern Sun. As illustrated in Figs. 4(c) and 5 (c), the photodissociation rates are increased by $220 \%$ for methane, $150 \%$ for acetylene and ethylene and $90 \%$ for carbon dioxide. Such enhanced photodissociation rates likely triggered a peculiar atmospheric chemistry in the early Earth's atmosphere, linked to the methane and more complex hydrocarbons increased photodissociation rates, which lead in turn to a more efficient formation of hydrocarbon aerosols. In our model, this formation relies exclusively on two reactions involving primary and secondary products of hydrocarbon photochemistry: ethynyl radical $\mathrm{C}_{2} \mathrm{H}$, acetylene $\mathrm{C}_{2} \mathrm{H}_{2}$ and allene $\mathrm{CH}_{2} \mathrm{CCH}_{2}$. These two reactions, the overall rate of which being displayed as double-dashed curves entitled "AER" in Figs. 4(c) and $5(\mathrm{c})$, are found to be more efficient in the Archean. In our model, the strong UV emission of the Sun at $3.5 \mathrm{Ga}$ enhances the organic haze formation rate by about $150 \%$.

The enhancement of the EUV emission of the Sun at $3.5 \mathrm{Ga}$ contributes even more importantly to the evolution of noble gases photoionization. As illustrated in Fig. 4(d) and $5(\mathrm{~d})$, helium, neon, argon and krypton photoionization rates are increased by about $1000 \%$ whereas xenon photoionization rate increases by about $4000 \%$. Such increase is due to the enhanced EUV part of the spectrum $(<$ $100 \mathrm{~nm}$ ) where the young Sun exhibits the most enhanced fluxes compared to the modern Sun (cf. Fig. 3). Moreover, in our model, the abundance of xenon in the ancient atmosphere is larger than in the modern one (Table 1) thus contributing to the particular increase of the xenon photodissociation rate in the Archean. Ionized nobles gases 

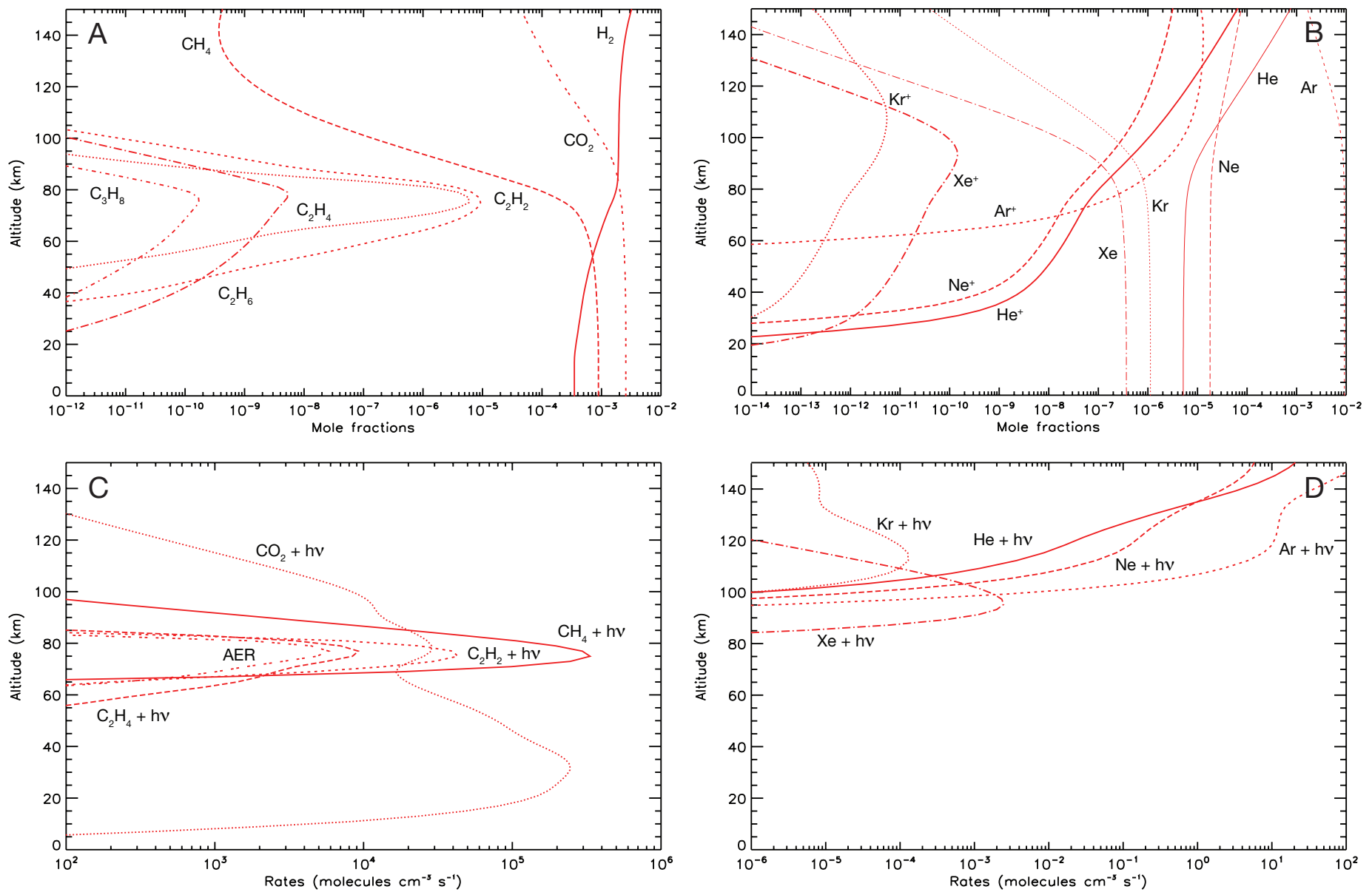

Figure 5: Results of the photochemical model with an incoming solar flux representative of the Sun at 3.5 Ga.

abundances - and particularly ionized xenon abundance are therefore more important in the Archean.

The column-integrated photoionization rates are normalized to the respective abundances of noble gases in Fig. 6 , to get rid of the variability of the different noble gases atmospheric abundances. The evolution of these rates as a function of time confirms that (i) noble gases were more efficiently photoionized under the ancient Sun than under the present Sun, and that (ii) xenon was more subject to photoionization than krypton and argon, but less than neon and helium.

\subsection{Vertical distribution of reaction rates}

The maximum production rate of organics by photochemistry takes place in an altitude range of approximately 60-100 km (Figs. 4 and 5). In this range, only xenon among noble gases is quantitatively photoionized as its photoionization rate takes place mainly in the 80-120 $\mathrm{km}$ range. Photoionization of neon, krypton and argon tends to occur at higher altitudes $(>100 \mathrm{~km})$. This general behavior can be explained in terms of their competiting opacity, each peaking at different altitudes. Therefore, if photoionized noble gases are prone to be trapped

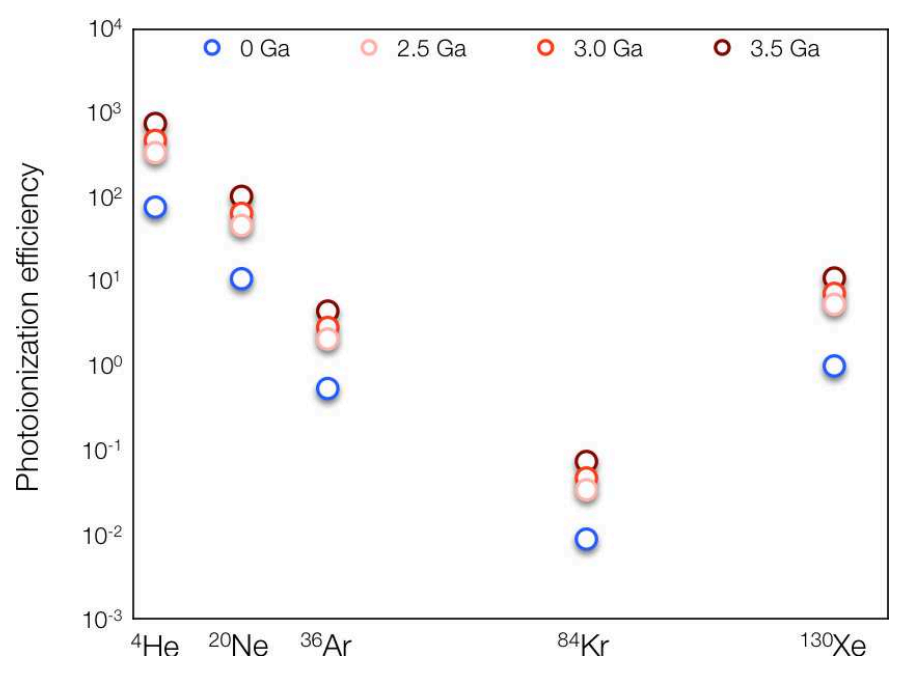

Figure 6: Noble gas photoionization rates, integrated over the whole atmospheric column, relative to the initial abundances of neutral nobles gases and normalized to the xenon photoionization rate under the present Sun. 


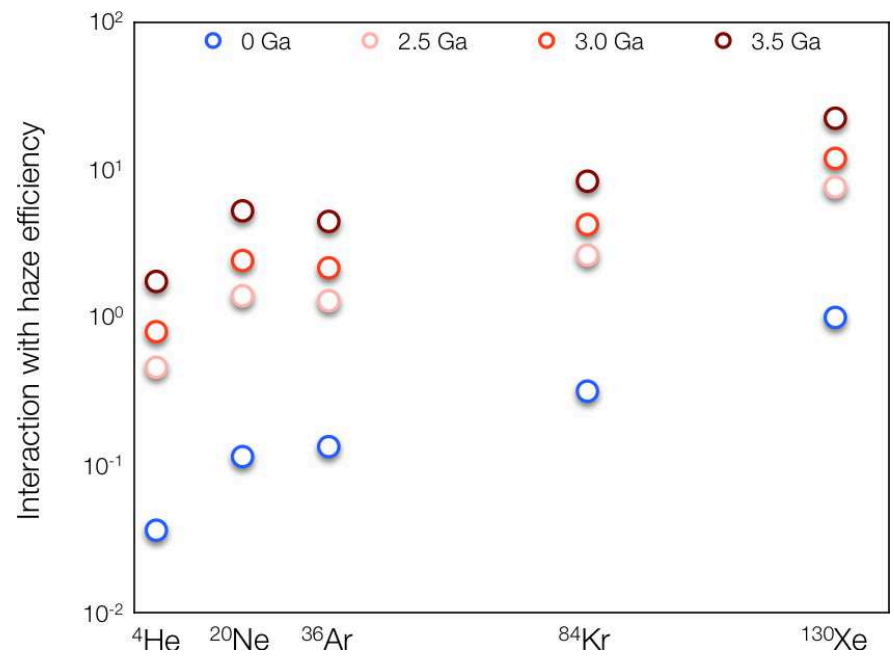

Figure 7: Cross-correlations between the haze formation rate and the noble gas photoionization rates, integrated over the whole atmospheric column and normalized to the xenon photoionization rate coefficient under the present Sun.

into growing organics, this photoionization rate distribution will tend to favour the interaction of ionized xenon with the organic haze relatively to the other ionized noble gases. This effect is illustrated in Fig. 7 which represents the integrated effect of cross correlating the organic haze formation rate and noble gas photoionization rate over the whole atmosphere. Such cross-correlation takes into account the different altitudes at which the reactions considered here take place. The evolution of these crosscorrelations as a function of time emphasizes the facts that (i) the photochemical production of organics and the photoionization of xenon peak at about the same altitude in our modelled atmosphere, that (ii) xenon was therefore more subject to interaction with an organic haze than all the other noble gases, and that (iii) such interaction was enhanced under the ancient Sun compared to the present Sun.

\subsection{Trapping of ionized noble gases into organic haze}

There is experimental evidence that ionized noble gases are efficiently trapped into growing organics (Marrocchi et al., 2011). Furthermore, several works have shown that (i) the yield of noble gas trapping into solids increases by orders of magnitude when noble gases are ionized compared to their neutral state, and (ii) trapped noble gases, when ionized, are mass-dependent isotopically fractionated, i.e., enriched in their heavy isotopes at the percent level. Two experiments are particularly relevant to this process. Frick et al. (1979) reported the results of an exhaustive investigation relative to different noble gases entrapment during Miller-Urey-like synthesis of carbonaceous, macromolecular, and kerogen-like substances. In particular, kerogen-like materials produced when applying an electric discharge to an artificial gas mixture contained high concentrations of noble gases trapped in the formed carbon-rich films that displayed strong elemental fractionation from their reservoirs. As pointed out in others subsequent investigations, noble gases photoionization is indeed responsible for establishing the observed noble gas patterns in the synthetic products but the underlying physicochemical mechanisms remain still to be unveiled (Bernatowicz and Fahey, 1986; Bernatowicz and Hagee, 1987; Ponganis et al., 1997; Hohenberg et al., 2002; Marrocchi et al., 2011). In a recent experiment, Marrocchi et al. (2011) evaporated kerogen and condensed it under ionizing conditions in a dilute xenon atmosphere, and observed a xenon isotopes enrichment of $1.3 \% \mathrm{u}^{-1}$ in the carbon condensate, which is comparable to the fractionation factor of $1.1 \% \mathrm{u}^{-1}$ expected here (Eqn. 1 and 2). In contrast, Marrocchi and Marty (2013) have recently shown that isotopic fractionation of neutral xenon during adsorption onto solids was indeed negiligbly small $(<0.02$ $\left.\% \mathrm{u}^{-1}\right)$.

As observed in laboratory experiments, the trapping properties of noble gases increases dramatically with their increasing mass (Table 2). Again, in presence of organic materials such as the photochemical haze, this process will favour the trapping of xenon over the other noble gases as, for instance its trapping efficiency is two orders of magnitude higher than that of argon, and marginally higher than that of krypton. However, we have shown previously that the different noble gases were not equally interacting with the organic haze over the whole atmosphere. Fig. 8 represents the overall efficiency of noble gas trapping into organic haze, that takes into account all processes involved, that is, the electronic properties of noble gases, the photochemical formation of organic haze, the vertical composition of our modelled atmosphere and the trapping properties of noble gases. The enhancement of the trapping efficiency of xenon over the other noble gases is clearly demonstrated. The second best-trapped noble gas is krypton, which is about a factor of 3 less efficiently trapped than xenon. It is therefore possible that the same process of isotopic fractionation and trapping could have affected as well atmospheric krypton which is also notably isotopically fractionated relative to chondritic krypton (by $0.5 \%$ $\mathrm{u}^{-1}$ ), to a less extent than xenon however. The evolution of these trapping efficiencies as a function of time emphasizes once again the fact that such interactions were enhanced under the ancient Sun compared to the present Sun.

Based on the coincidence of peaks for photochemical production of organics and the enhanced photoionization of xenon in our modelled atmosphere, we propose that the formation of an organic haze in the Archean atmosphere

Table 2: Noble gases trapping properties for electron discharge kerogen (calculated from Frick et al., 1979, Table 1)

\begin{tabular}{ccccc}
\hline $\mathrm{He}$ & $\mathrm{Ne}$ & $\mathrm{Ar}$ & $\mathrm{Kr}$ & $\mathrm{Xe}$ \\
$2.26 \times 10^{-4}$ & $5.65 \times 10^{-4}$ & $1.20 \times 10^{-4}$ & $1.61 \times 10^{-2}$ & $1.84 \times 10^{-2}$ \\
\hline (in ccSTP g & & & &
\end{tabular}




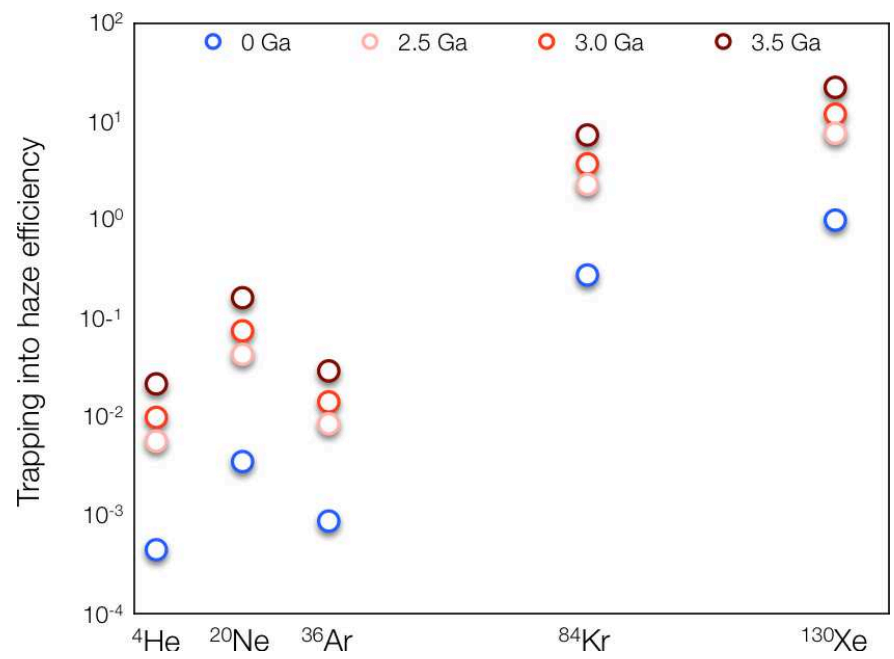

Figure 8: Noble gases trapping properties, integrated over the whole atmospheric column and normalized to the xenon trapping properties under the present Sun.

provided an efficient trap for the atmospheric xenon ionized by the enhanced flux of EUV from the younger Sun. This mechanism provides a way to isolate in a solid phase a xenon component enriched in its heavy isotopes, as is the present-day atmospheric xenon (with respect to cosmochemical xenon). As a consequence, to be able to explain the xenon paradox, atmospheric xenon (not trapped in the organic haze) had to be lost into space, the haze acting only as a temporary trap of isotopically "heavy" xenon that tends to concentrate heavy xenon isotopes in the lower part $(<120 \mathrm{~km})$ of the atmosphere. We therefore envision a distillation process during which xenon is continuously lost from the atmosphere into space by a nonthermal escape process, with only a tiny fraction, enriched in heavy isotopes, being temporarily stored in organics. The cumulative effect of such process will result into progressive depletion of atmospheric xenon together with its enrichment in its heavy isotopes, as observed in the modern atmosphere.

Our model does not resolve entirely the xenon paradox since one needs to find a suitable non-thermal process to loose atmospheric xenon preferentially to other noble gases. One possibility is that photoionized xenon indeed escaped as an ion, probably in polar winds of hydrogen atoms $\mathrm{H}$ and hydrogen ions $\mathrm{H}^{+}$channelled by open planetary magnetic field lines. Since xenon, alone among the noble gases, is more easily ionized than hydrogen, it will tend to stay so in this hydrodynamic $\mathrm{H}_{-} \mathrm{H}^{+}$wind and be dragged by the strong resulting Coulomb interactions, whilst the other noble gases would get quickly neutralized and unable to follow. Under these circumstances, hydrodynamic escape could apply uniquely to xenon among the noble gases over a wide range of hydrogen escape fluxes (Zahnle, 2011). And since both hydrodynamic escape and photoionizations are fueled by solar EUV radiation, this mechanism would have been all the more efficient as the Sun was younger. In such a scheme the combination of atmospheric escape $(\sim 500 \mathrm{~km})$ and temporal trapping into the organic haze at a lower altitude $(<120 \mathrm{~km})$ could have resulted in the overall concentration of heavy xenon isotopes. It may also be that xenon escape could have further fractionated its isotopes in the same way, that is, preferential retention of heavy xenon isotopes in the atmosphere over time. Investigating properly the combined effects of these two processes on the xenon elemental and isotopic compositions will require to develop a quantitative model for xenon trapping into organics and its escape from the atmosphere.

If our approach is correct, however, it leads to several important planetary implications. Firstly, our model implies that the isotopic composition of xenon of the ancient atmosphere may provide independent evidence for the existence of an organic haze in the Archean atmosphere. Secondly, since the atmosphere of Mars also presents a xenon paradox comparable (but not exactly similar in term of isotopic fractionation) to the terrestrial one, our model suggests that the ancient atmosphere of Mars might have also hosted an organic haze, therefore rising the question of a source of methane on Mars. Thirdly, our model predicts that xenon in the atmosphere of Titan, which is effectively highly depleted as are the other noble gases (Niemann et al., 2005), should also be isotopically fractionated to an extent exceeding probably the case of terrestrial xenon. This inference is subject to be tested when a sufficiently sensitive dedicated analytical system will be launched to Titan.

\section{Conclusion}

The evolution of the isotopic composition of xenon in the atmosphere over time has been re-evaluated in the light of the enhanced UV emission of the young Sun and of the peculiar atmospheric chemistry that such enhancement presumably triggered. We interpret the "xenon paradox" as resulting from its efficient photoionization by hard UV light incoming into the early atmosphere, which triggers an efficient trapping of its heavier isotopes within a primitive organic haze as well as its preferential escape compared to other atmospheric volatiles. Most of the ionized xenon remaining in the atmosphere would have then escaped into space by hydrodynamic escape, even if it still requires us to figure out how to keep the xenon ionized long enough.

\section{Acknowledgement}

This project was funded by the European Research Council under the European Community's Seventh Framework Program (FP7/20072013 grant agreement no. 267255 to BM). We appreciated discussions with Kevin Zahnle and James Lyons. CRPG contribution \#XXX (to be added later on if the paper accepted) 
Allègre, C.J., Staudacher, T., Sarda, P., Kurz, M., 1983. Constraints on evolution of Earth's mantle from rare gas systematics. Nature 303, 762-766.

Basford, J.R., Dragon, J.C., Pepin, R.O., Coscio, Jr., M.R., Murthy, V.R., 1973. Krypton and xenon in lunar fines. Proc. Lunar Sci. Conf. 4, 1915-1955.

Bernatowicz, T.J., Fahey, A.J., 1986. Xe isotopic fractionation in a cathodeless glow discharge. Geochim. Cosmochim. Acta 50, 445452 .

Bernatowicz, T.J., Hagee, B.E., 1987. Isotopic fractionation of $\mathrm{Kr}$ and Xe implanted in solids at very low energies. Geochim. Cosmochim. Acta 51, 1599-1611.

Chan, W.F., Cooper, G., Brion, C.E., 1991. Absolute optical oscillator strengths for the electronic excitation of atoms at high resolution: Experimental methods and measurements for helium. Phys. Rev. A 44, 186-204.

Chan, W.F., Cooper, G., Guo, X., Brion, C.E., 1992a. Absolute optical oscillator strengths for the electronic excitation of atoms at high resolution. II. The photoabsorption of neon. Phys. Rev. A $45,1420-1433$.

Chan, W.F., Cooper, G., Guo, X., Burton, G.R., Brion, C.E., 1992b. Absolute optical oscillator strengths for the electronic excitation of atoms at high resolution. III. The photoabsorption of argon, krypton, and xenon. Phys. Rev. A 46, 149-171.

Claire, M.W., Sheets, J., Cohen, M., Ribas, I., Meadows, V.S., Catling, D.C., 2012. The evolution of solar flux from $0.1 \mathrm{~nm}$ to $160 \mu \mathrm{m}$ : Quantitative estimates for planetary studies. Astrophys. J. 757,95 .

Dauphas, N., 2003. The dual origin of the terrestrial atmosphere. Icarus $165,326-339$.

Domagal-Goldman, S.D., Kasting, J.F., Johnston, D.T., Farquhar, J., 2008. Organic haze, glaciations and multiple sulfur isotopes in the Mid-Archean Era. Earth Planet. Sci. Lett. 269, 29-40.

Farquhar, J., Bao, H., Thiemens, M., 2000. Amospheric influence of Earth's earliest sulfur cycle. Science 289, 756-758.

Farquhar, J., Wing, B.A., 2003. Multiple sulfur isotopes and the evolution of the atmosphere. Earth Planet. Sci. Lett. 213, 1-13.

Feulner, G., 2012. The faint young Sun problem. Rev. Geophys. 50, RG2006.

Frick, U., Mack, R., Chang, S., 1979. Noble gas trapping and fractionation during synthesis of carbonaceous matter, pp. 1961-1972.

Hébrard, E., Dobrijevic, M., Loison, J.C., Bergeat, A., Hickson, K.M., 2012. Neutral production of hydrogen isocyanide (HNC) and hydrogen cyanide (HCN) in Titan's upper atmosphere. Astron. Astrophys. 541, A21.

Hindmarsh, A.C., 1983. ODEPACK, a systematized collection of ODE solvers.

Hohenberg, C.M., Thonnard, N., Meshik, A., 2002. Active capture and anomalous adsorption: new mechanisms for the incorporation of heavy noble gases. Meteorit. Planet. Sci. 37, 257-267.

Holland, G., Sherwood Lollar, B., Li, L., Lacrampe-Couloume, G., Slater, G.F., Ballentine, C.J., 2013. Deep fracture fluids isolated in the crust since the Precambrian era. Nature 497, 357-360.

Kasting, J.F., 2010. Early Earth: Faint young Sun redux. Nature 464, 687-689.

Kasting, J.F., Zahnle, K.J., Walker, J.C.G., 1983. Photochemistry of methane in the Earth's early atmosphere, in: B. Nagy, R. Weber, J.G., Schidlowski, M. (Eds.), Developments and Interactions of the Precambrian Atmosphere, Lithosphere and Biosphere. Elsevier. Volume 7 of Developments in Precambrian Geology, pp. 13-40.

Kharecha, P., Kasting, J.F., Siefert, J., 2005. A coupled atmosphereecosystem model of the early Archean Earth. Geobiology 3,5376 .

Lee, J.Y., Marti, K., Severinghaus, J.P., Kawamura, K., Yoo, H.S., Lee, J.B., Kim, J.S., 2006. A redetermination of the isotopic abundances of atmospheric Ar. Geochim. Cosmochim. Ac. 70, 4507-4512

Lide, D.R. (Ed.), 2009. CRC Handbook of Chemistry and Physics. CRC Press; $90^{\text {th }}$ edition.

Lokhov, K., Levsky, K., Begemann, F., 2002. Volatile components in Karelian shungites as indicators of composition of Protherozoic atmosphere, in: Astrobiology Expeditions.

Marrocchi, Y., Marty, B., 2013. Experimental determination of the xenon isotopic fractionation during adsorption. Geophys. Res. Lett. 40, 4165-4170.

Marrocchi, Y., Marty, B., Reinhardt, P., Robert, F., 2011. Adsorption of xenon ions onto defects in organic surfaces: Implications for the origin and the nature of organics in primitive meteorites. Geochim. Cosmochim. Ac. 75, 6255-6266.

Marty, B., 2012. The origins and concentrations of water, carbon, nitrogen and noble gases on Earth. Earth Planet. Sci. Lett. 313$314,56-66$

Massie, S.T., Hunten, D.M., 1981. Stratospheric eddy diffusion coefficients from tracer data. J. Geophys. Res. - Oc. Atm. 86, 9859 9868.

Meshik, A.P., Hohenberg, C.M., Pravdivtseva, O.V., Kapusta, Y.S., 2001. Weak decay of ${ }^{130} \mathrm{Ba}$ and ${ }^{132} \mathrm{Ba}$ : Geochemical measurements. Phys. Rev. C 64, 035205.

Niemann, H. and 17 co-authors, 2005. The abundances of constituents of Titan's atmosphere from the GCMS instrument on the Huygens probe. Nature 438, 779-784.

Owen, T., Bar-Nun, A., Kleinfeld, I., 1992. Possible cometary origin of heavy noble gases in the atmospheres of Venus, Earth and Mars. Nature 358, 43-46.

Ozima, M., Podosek, F.A., 2002. Noble gas geochemistry, Second edition. 286 p.. Cambridge University Press, Cambridge.

Pavlov, A.A., Brown, L.L., Kasting, J., 2001a. UV shielding of $\mathrm{NH}_{3}$ and $\mathrm{O}_{2}$ by organic hazes in the Archean atmosphere. J. Geophys. Res. - Planet. 106, 23,267-23,287.

Pavlov, A.A., Kasting, J.F., 2002. Mass-independent fractionation of sulfur isotopes in Archean sediments: Strong evidence for an anoxic Archean atmosphere. Astrobiology 2, 27-41.

Pavlov, A.A., Kasting, J.F., Eigenbrode, J.L., Freeman, K.H., 2001b. Organic haze in Earth's early atmosphere: Source of low- ${ }^{13} \mathrm{C}$ Late Archean kerogens? Geology 29, 1003-1006.

Pepin, R.O., 1991. On the origin and early evolution of terrestrial planet atmospheres and meteoritic volatiles. Icarus 92, 2-79.

Pepin, R.O., Porcelli, D., 2006. Xenon isotope systematics, giant impacts, and mantle degassing on the early Earth. Earth Planet. Sci. Lett. 250, 470-485.

Ponganis, K., Graf, T., Marti, K., 1997. Isotopic fractionation in lowenergy ion implantation. J. Geophys. Res. - Planet. 102, 1933519343.

Powner, M.W., Gerland, B., Sutherland, J.D., 2009. Synthesis of activated pyrimidine ribonucleotides in prebiotically plausible conditions. Nature 459, 239-242.

Pujol, M., Marty, B., Burgess, R., 2011. Chondritic-like xenon trapped in Archean rocks: A possible signature of the ancient atmosphere. Earth Planet. Sci. Lett. 308, 298-301.

Pujol, M., Marty, B., Burgess, R., Turner, G., Philippot, P., 2013. Argon isotopic composition of Archaean atmosphere probes early Earth geodynamics. Nature 498, 87-90.

Pujol, M., Marty, B., Burnard, P., Philippot, P., 2009. Xenon in Archean barite: Weak decay of ${ }^{130} \mathrm{Ba}$, mass-dependent isotopic fractionation and implication for barite formation. Geochim. Cosmochim. Acta 73, 6834-6846.

Ribas, I., Guinan, E.F., Güdel, M., Audard, M., 2005. Evolution of the solar activity over time and effects on planetary atmospheres. I. High-energy irradiances (1-1700 Å). Astrophys. J. 622, 680-694.

Ribas, I., Porto de Mello, G.F., Ferreira, L.D., Selsis, F., Hébrard, E., Catalán, S., Garcés, A., Nascimento Jr., J.D., de Medeiros, J.R., 2010. Evolution of the solar activity over time and effects on planetary atmospheres. II. $\kappa^{1}$ Ceti as an analog of the Sun when Life arose on Earth. Astrophys. J. 714, 384-395.

Rosing, M.T., Bird, D.K., Sleep, N.H., Bjerrum, C.J., 2010. No climate paradox under the faint early Sun. Nature 464, 744-747.

Rye, R., Kuo, P.H., Holland, H.D., 1995. Atmospheric carbon dioxide concentrations before 2.2 billion years ago. Nature 378, 603-605.

Sagan, C., Chyba, C., 1999. The early Faint Sun paradox: Organic shielding of ultraviolet-labile greenhouse gases. Science 276, 1217 1221 . 
Sanloup, C., Bonev, S.A., Hochlaf, M., Maynard-Casely, H.E., 2013. Reactivity of xenon with ice at planetary conditions. Phys. Rev. Lett. 110, 265501.

Sanloup, C., Schmidt, B.C., Perez, E.M.C., Jambon, A., Gregoryanz, E., Mezouar, M., 2005. Retention of xenon in quartz and earth's missing xenon. Science 310, 1174-1177.

Shcheka, S.S., Keppler, H., 2012. The origin of the terrestrial noblegas signature. Nature 490, 531-534.

Som, S.M., Catling, D.C., Harnmeijer, J.P., Polivka, P.M., Buick, R., 2012. Air density 2.7 billion years ago limited to less than twice modern levels by fossil raindrop imprints. Nature 484, 359-362.

Srinivasan, B., 1976. Barites: Anomalous xenon from spalation and neutron-induced reactions. Earth Planet. Sci. Lett. 31, 129141.

Tian, F., Kasting, J.F., Zahnle, K., 2011. Revisiting HCN formation in Earth's early atmosphere. Earth Planet. Sci. Lett. 308, 417-423.

Tolstikhin, I.N., Marty, B., 1998. The evolution of terrestrial volatiles: a view from helium, neon, argon and nitrogen isotope modelling. Chem. Geol. 147, 27-52.

Trainer, M.G., Pavlov, A.A., Curtis, D.B., McKay, Christopher P. Worsnop, D.R., Delia, A.E., Toohey, D.W., Toon, O.B., Tolbert, M.A., 2004. Haze aerosols in the atmosphere of early Earth: Manna from Heaven. Astrobiology 4, 409-419.

Trainer, M.G., Pavlov, A.A., Dewitt, H.L., Jimenez, J.L., McKay, C.P., Toon, O.B., Tolbert, M.A., 2006. Organic haze on Titan and the early Earth. Proc. Natl. Acad. Sci. 103, 18035-18042.

Walker, J.C.G., 1977. Evolution of the atmosphere. 318 p.. Macmillan, New York.

Wieler, R., Baur, H., 1994. Krypton and xenon from the solar wind and solar energetic particles in two lunar ilmenites of different antiquity. Meteoritics 29, 570-580.

Zahnle, K., 1986. Photochemistry of methane and the formation of hydrocyanic acid (HCN) in the Earth's early atmosphere. J. Geophys. Res. - Atmos. 91, 2819-2834.

Zahnle, K., 2011. Xenon the Magnificent. Mineral. Mag. 75, 2241.

Zartman, R.E., Wasserburg, G.J., Reynolds, J.H., 1961. Helium, argon, and carbon in some natural gases. J. Geophys. Res. 66, $277-306$.

Zerkle, A.L., Claire, M.W., Domagal-Goldman, S.D., Farquahr, J. Poulton, S.W., 2012. A bistable organic-rich atmosphere on the Neoarchaean Earth. Nat. Geosci. 5, 359-363.

Zhu, Q., Jung, D.Y., Oganov, A.R., Glass, C.W., Gatti, C., Lyakhov, A.O., 2013. Stability of xenon oxides at high pressures. Nature Chem. 5, 61-65. 